\title{
Association of cause of injury and traumatic axonal injury: a clinical MRI study of moderate and severe traumatic brain injury
}

\author{
*Hans Kristian Moe, MD, ${ }^{1}$ Janne Limandvik Myhr, MD, ${ }^{1}$ Kent Gøran Moen, MD, PhD, ${ }^{1,2}$ \\ Asta Kristine Håberg, MD, PhD, , Toril Skandsen, MD, PhD, ${ }^{1,4}$ and Anne Vik, MD, PhD ${ }^{1,5}$ \\ 'Department of Neuromedicine and Movement Science, Norwegian University of Science and Technology (NTNU), Trondheim; \\ 2Department of Radiology, Nord-Trøndelag Hospital Trust, Levanger; and Departments of ${ }^{3}$ Radiology and Nuclear Medicine, \\ ${ }^{4}$ Physical Medicine and Rehabilitation, and ${ }^{5}$ Neurosurgery, St. Olavs Hospital, Trondheim University Hospital, Trondheim, \\ Norway
}

OBJECTIVE The authors investigated the association between the cause of injury and the occurrence and grade of traumatic axonal injury (TAI) on clinical MRI in patients with moderate or severe traumatic brain injury (TBI).

METHODS Data for a total of 396 consecutive patients, aged 7-70 years, with moderate or severe TBI admitted to a level 1 trauma center were prospectively registered. Data were included for analysis from the 219 patients who had MRI performed within 35 days (median 8, IQR 4-17 days) and for whom cause of injury was known. Cause of injury was registered as road traffic accident (RTA) or fall (both with respective subcategories), alpine skiing or snowboarding accident, or violence. The MRI protocol consisted of T2*-weighted gradient echo, FLAIR, and diffusion-weighted imaging scans. TAl lesions were evaluated in a blinded manner and categorized into 3 grades, hemispheric/cerebellar white matter (grade 1), corpus callosum (grade 2), and brainstem (grade 3). The absence of TAI was analyzed as grade 0 . Contusions and mass lesions on CT were also registered.

RESULTS Cause of injury did not differ between included and nonincluded patients. TAI was found in $83 \%$ of patients in the included group after RTAs and $62 \%$ after falls $(p<0.001)$. Observed TAl grades differed between the subcategories of both RTAs $(p=0.004)$ and falls $(p=0.006)$. Pedestrians in RTAs, car drivers/passengers in RTAs, and alpine skiers had the highest prevalence of TAI (89\%-100\%) and the highest TAI grades (70\%-82\% TAI grades $2-3)$. TAI was found in $76 \%$ of patients after falls from > own height (45\% TAI grade $2-3$ ), $63 \%$ after falls down the stairs $(26 \%$ TAl grade $2-3$ ), and $31 \%$ after falls from $\leq$ own height (12\% TAl grade $2-3)$. Moreover, $53 \%$ of patients with TAl after RTAs and $68 \%$ with TAl after falls had cortical contusions or mass lesions on CT.

CONCLUSIONS This prospective study of moderate and severe TBI is to the authors' knowledge the first clinical MRI study to demonstrate both the high prevalence and grade of TAl after most of the different types of RTAs, alpine skiing accidents, and falls from a height. Importantly, TAI was also common following more low-energy trauma such as falls down the stairs or from own height. Physicians managing TBI patients in the acute phase should be aware of the possibility of TAI no matter the cause of injury and also when the CT scan shows cortical contusions or mass lesions.

https://thejns.org/doi/abs/10.3171/2019.6.JNS191040

KEYWORDS neuroimaging; diffuse axonal injury; accidents; craniocerebral trauma; magnetic resonance imaging; traumatic brain injury; diagnostic technique

$\mathrm{T}$ RAUMATIC axonal injury (TAI), or diffuse axonal injury, is a major cause of disability following traumatic brain injury (TBI). $)^{2,5,33}$ TAI occurs when angular and/or rotational acceleration and deceleration forces cause deformation and stretching of the brain tis- sue. ${ }^{10,16}$ Axonal stretch disrupts the normal function of the axolemma, which in turn interferes with the integrity of the axon and can result in axonal varicosities and often secondary axotomy. ${ }^{16,19}$

The gold standard for diagnosing TAI is through his-

ABBREVIATIONS DWI = diffusion-weighted imaging; GCS = Glasgow Coma Scale; GOSE = Glasgow Outcome Scale Extended; GRE = gradient echo; HISS = Head Injury Severity Scale; ISS = Injury Severity Score; RTA = road traffic accident; SWI = susceptibility-weighted imaging; TAI = traumatic axonal injury; TBI = traumatic brain injury. SUBMITTED April 12, 2019. ACCEPTED June 25, 2019.

INCLUDE WHEN CITING Published online October 11, 2019; DOI: 10.3171/2019.6.JNS191040.

* H.K.M. and J.L.M. contributed equally to this work. 
topathological examination, but indirect signs of these lesions can be found on medical imaging in surviving patients. ${ }^{11}$ Although CT remains the imaging modality of choice in the acute setting after TBI, it grossly underestimates the occurrence of TAI. ${ }^{24,27}$ However, the focal edema and microhemorrhages which frequently accompany TAI can be detected by clinical MRI ${ }^{30,36}$ These MRI lesions were found to have the same distribution as the TAI lesions described histologically by Adams et al. and Gennarelli et al.2,10,12 Recent studies have also shown that TAI lesions on clinical MRI are associated with disrupted white matter microstructure on diffusion tensor imaging, ${ }^{26,35}$ as well as with histopathological findings of TAI postmortem demonstrated in a case report. ${ }^{22}$ Adams et al. originally graded TAI based on autopsy studies, ${ }^{2}$ and Gentry et al. ${ }^{12}$ later described a modified grading based on MRI: grade 1, lesions in the hemispheric and/or cerebellar white matter; grade 2, lesions in the corpus callosum; and grade 3 , lesions in the brainstem. It is assumed that there is successive involvement of more central and deeper brain structures as the impact load increases. ${ }^{28,33}$ Hence, the TAI grades are assumed to reflect the energy of the trauma. Furthermore, MRI studies have shown that TAI lesions in the thalamus and brainstem, and especially bilateral lesions, are associated with both low Glasgow Coma Scale (GCS) scores in the acute phase and worse global outcome in the long run. ${ }^{24,32}$

Not surprisingly, TAI is most often found in patients who have suffered high-energy trauma, such as road traffic accidents (RTAs) ${ }^{4,6,15,33}$ and falls from considerable heights. ${ }^{3,4}$ However, there is now increasing evidence of TAI in all severities of TBI, ${ }^{17,33}$ indicating that TAI may also be an important type of injury after low-energy trauma. Some histopathological studies in the field exist, ${ }^{3,6}$ but to our knowledge there are no previous studies using clinical neuroimaging to thoroughly explore the relation between cause of injury and TAI in patients surviving the acute phase.

Hence, the primary aim of this study was to investigate how the different causes of injury relate to the occurrence and grade of TAI on clinical MRI in a cohort of moderate and severe TBI patients at a level 1 trauma center. The secondary aim was to explore how the cause of injury relates to findings of contusions and mass lesions on the CT scan in patients with TAI.

\section{Methods \\ Patients}

Between October 2004 and October 2014, all patients admitted to Trondheim University Hospital (level 1 trauma center) with moderate or severe head injury according to the Head Injury Severity Scale ${ }^{34}$ (HISS) were prospectively registered in the Trondheim TBI studies. Of the 396 patients (age 7-70 years), 219 patients had both known cause of injury and MRI within 5 weeks of the trauma and were included in this study (Fig. 1). The remaining 177 nonincluded patients are presented and compared with the included patients with regard to patient characteristics and cause of injury, but they were not analyzed with regard to the research questions and their TAI status was unknown.

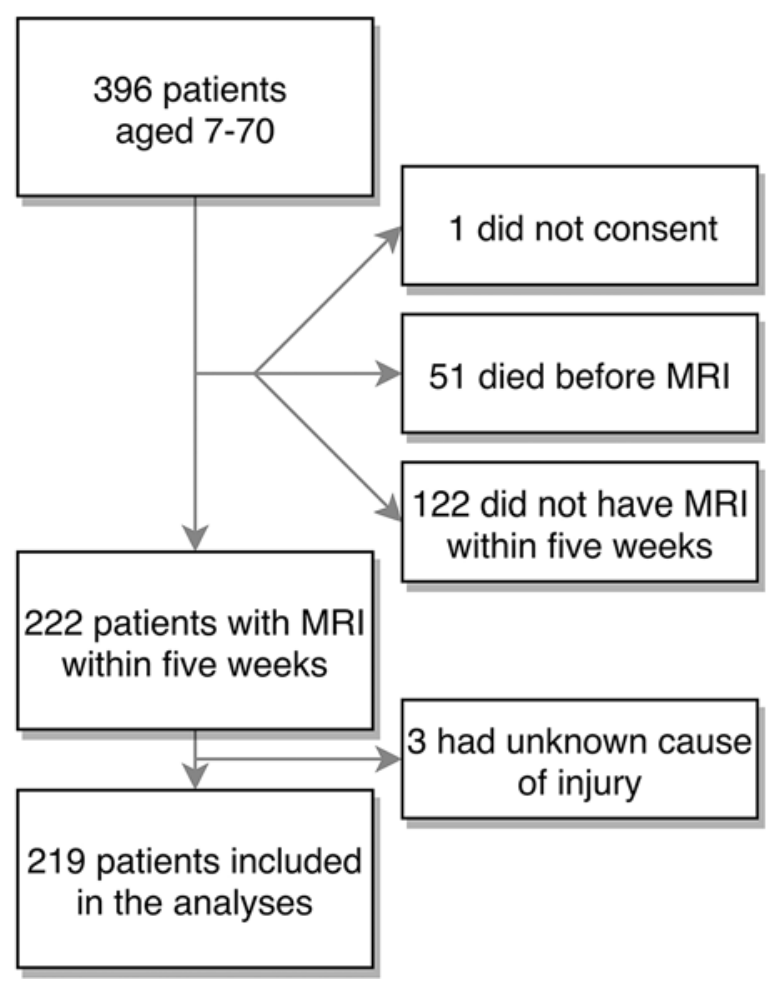

FIG. 1. Flowchart of the consecutive TBI cohort and the 219 patients included in this MRI study.

\section{Cause of Injury}

The cause of injury was prospectively collected as part of the research database and registered as one of the following: RTA, fall, alpine skiing accident (which also included snowboarding accidents), violence, or other (e.g., horseback riding accident). RTAs were divided into 4 subcategories: pedestrian hit by motorized vehicle, car driver/ passenger, motorcyclist (motorcycle/moped/snowmobile), and bicyclist. Fall height was divided into 7 subcategories: $\geq 5 \mathrm{~m},>2 \mathrm{~m}$ but $<5 \mathrm{~m},>$ own height but $\leq 2 \mathrm{~m}$, fall down the stairs, fall from own height standing upright, fall from $<$ own height (e.g., fall from sitting in a chair), and fall from unknown height. For some of the analyses we combined several fall subcategories into the following categories: fall from $>$ own height, fall down the stairs, and fall from $\leq$ own height.

\section{Other Injury Variables}

Other injury-related variables were obtained from the prospective database, including GCS score before intubation or at admission for nonintubated patients, intubation status, secondary events in the form of hypoxia $\left(\mathrm{O}_{2}\right.$ saturation below 92\%) and hypotension (systolic blood pressure $<90 \mathrm{~mm} \mathrm{Hg}$ ) before or at admission, and pupillary dilatation at admission. Injury Severity Score (ISS) was estimated for all patients as a measure of overall injury. A radiologist or resident in neurosurgery/radiology in collaboration with 3 consultants in neuroradiology reviewed all admission CT scans for the following findings: 1) significant mass lesions (hematoma or contusion, defined as either evacuated lesions, or nonevacuated lesions $>25$ 
$\mathrm{cm}^{3}$, i.e., Marshall CT score 5 or 6), ${ }^{23}$ and 2) the presence of cortical contusions. In-hospital mortality was also registered. Brain injury-related outcome was assessed 12 months postinjury by use of the Glasgow Outcome Scale Extended (GOSE). ${ }^{18,33}$ Ten patients were lost to follow-up, while for an additional 5 patients the scores at 6 months were used due to missing assessment at 12 months.

\section{Clinical MRI}

The clinical MRI was performed using a harmonized protocol $^{25,33}$ (at the study hospital or at one of the local hospitals in the region) with either a 1.5-T (Siemens Symphony, Siemens Avanto, Philips Gyroscan NT Intera) or a 3-T (Siemens Skyra $[n=6]$ ) system. The protocol consisted of 5 different imaging sequences: 1) sagittal turbo spin echo T2-weighted imaging; 2) sagittal, transverse, and coronal T2-weighted FLAIR imaging; 3) transverse T2*weighted gradient echo (GRE) imaging or susceptibilityweighted imaging (SWI $[\mathrm{n}=15]$ ); 4) transverse spin echo T1-weighted imaging; and 5) diffusion-weighted imaging (DWI).

\section{Image Analyses}

The MR images were read based on the sequences FLAIR, T2*GRE/SWI, and DWI. All readers were blinded to patient identification, clinical information, and time of examination. A resident in radiology (K.G.M.), in close cooperation with experienced neuroradiologists (K.A.K. and J.R.), read 153 of the MR images. In a previous study, a third neuroradiologist (M.F.) rescored 89 of these scans for interrater analyses, and the reliability for TAI grading was found to be good (linear Cohen's $x$ 0.74). ${ }^{25}$ The remaining $66 \mathrm{MR}$ images were read by a resident in radiology (J.X.) and the final score was reached on consensus between him and either K.A.K. or M.F.

The presence of TAI in the hemispheres/cerebellum was recorded as TAI grade 1, in the corpus callosum as TAI grade 2, and in the brainstem as TAI grade 3. Although not part of the grading scale, the presence of TAI in the thalamus was also recorded. In addition, bilateral TAIs were recorded for both the brainstem and thalamus. TAI was differentiated from cortical contusion on the basis of 2 characteristics: 1) location: the bulk of the contusional lesion is more often located in the cerebral cortex, i.e., close to the skull, whereas that of a TAI lesion is more often situated in the hemispheric gray and white matter junction; and 2) morphology: microhemorrhages on T2*GRE or SWI strengthen the likelihood of TAI, whereas bone edema or bone injuries favor contusions. Traumatic parenchymal hemorrhage (identified by large hemorrhagic components on FLAIR) and iatrogenic injuries from insertion of intracranial pressure monitoring devices or external ventricular drains were not counted as TAIs. White matter hyperintensities (WMHs) not interpreted as trauma related were considered nonspecific and consequently not recorded as TAIs..$^{24}$ Information from all available MRI sequences were used to identify all types of lesions.

\section{Statistical Analyses}

Patient and injury variables are listed as counts with percentages and medians with interquartile ranges (IQRs). The Kruskal-Wallis test and Mann-Whitney U-test were used for group comparisons of all continuous and ordinal variables. Chi-square and Fisher's exact tests were used for comparison of proportions. The absence of TAI was analyzed as TAI grade 0 . TAI grades among different groups of patients were presented as medians with IQRs. Differences in TAI grades related to the subcategories of cause of injury were analyzed with the Kruskal-Wallis test (subcategory "unknown fall height" not included in the analysis). The predictive value of TAI grade on functional outcome was analyzed using multiple binary logistic regression in order to adjust for known outcome predictors. ${ }^{21}$ The outcome variable (GOSE score) was dichotomized into poor outcome (scores 1-4) and favorable outcome (scores 5-8) and set as the dependent variable. TAI grade, patient age, and GCS score were analyzed as covariates, while mass lesions, cortical contusions, and pupillary dilatation were analyzed as factors. Statistical significance was set to $\mathrm{p}<0.05$ (two-sided). Data were analyzed using RStudio 1.1.456.

\section{Ethics}

The study was approved by the Regional Committee for Medical Research Ethics. Written informed consent was given by surviving patients or, for incapacitated or underage individuals, their next of kin. The Norwegian Directorate of Health approved use of data from deceased individuals.

\section{Results}

\section{Demographics and Cause of Injury}

There were no significant differences in the cause of injury between the 219 included patients and the 170 nonincluded patients with known cause of injury. The nonincluded patients were older and had more severe injuries and higher in-hospital mortality than the included patients (Table 1). Among the included patients, RTA was the most frequent cause of injury (50\%, Table 1$)$. All pedestrians and 3 of the bicyclists were hit by a car. A total of $37 \%$ of the patients were injured in falls, $5 \%$ in alpine skiing accidents, and $4 \%$ by violence. The distribution of patients in the subcategories of RTAs and falls is shown in Fig. 2.

\section{Cause of Injury and Occurrence of TAI on Clinical MRI}

Clinical MRI was performed at a median of 8 days postinjury (IQR 4-17 days). The time to MRI was no different after RTAs than after falls $(\mathrm{p}=0.461)$. A total of $74 \%$ of the included patients had TAI. Among those with TAI, age differed significantly between those injured in RTAs and those injured in falls $(\mathrm{p}=0.020)$, while GCS scores did not $(\mathrm{p}=0.104$, Table 2$)$.

In the RTA category, $83 \%$ had TAI (100\% of pedestrians, $89 \%$ of car drivers/passengers, and $68 \%$ of bicyclists). In the fall category, $62 \%$ had TAI, a significantly lower proportion than for the RTA category ( $p<0.001)$. TAI was found among $76 \%$ of patients who fell from $>$ own height, $63 \%$ of patients who fell down the stairs, and $31 \%$ of patients who fell from $\leq$ own height. Seventy-three percent of patients who fell $>2 \mathrm{~m}$ had TAI compared to $50 \%$ for 
TABLE 1. Patient characteristics, cause of injury, and injury-related variables in the included and nonincluded patients in the consecutive moderate or severe TBI cohort

\begin{tabular}{|c|c|c|c|}
\hline Variable & $\begin{array}{l}\text { Included Pts } \\
(n=219)\end{array}$ & $\begin{array}{l}\text { Nonincluded } \\
\text { Pts }(n=177)\end{array}$ & $\begin{array}{c}p \\
\text { Value }\end{array}$ \\
\hline Age in yrs & $29(20-46)$ & $44(25-56)$ & $<0.001$ \\
\hline Sex & & & 0.956 \\
\hline Male & $169(77)$ & $137(77)$ & \\
\hline Female & $50(23)$ & $40(23)$ & \\
\hline \multicolumn{4}{|l|}{ Cause of injury* } \\
\hline RTA & $109(50)$ & $75(44)$ & 0.268 \\
\hline Fall & $81(37)$ & $76(45)$ & 0.124 \\
\hline Violence & $9(4)$ & $6(4)$ & 0.768 \\
\hline Alpine skiing† & $10(5)$ & $2(1)$ & 0.075 \\
\hline Otherf & $10(5)$ & $11(6)$ & 0.410 \\
\hline GCS score & $9(6-12)$ & $7(3-13)$ & 0.040 \\
\hline Severe TBI§ & $98(46)$ & $97(58)$ & 0.022 \\
\hline Intubation & $150(69)$ & $122(69)$ & 0.913 \\
\hline Hypoxia and/or hypotensionף & $45(21)$ & $61(35)$ & 0.002 \\
\hline \multicolumn{4}{|l|}{ Pupil dilatation at admission } \\
\hline Unilateral & $19(9)$ & $28(17)$ & 0.018 \\
\hline Bilateral & $6(3)$ & $25(15)$ & $<0.001$ \\
\hline ISS & $25(17-33)$ & $25(17-4)$ & 0.627 \\
\hline Rotterdam CT score & $3(2-3)$ & $3(2-4)$ & 0.004 \\
\hline Evacuated mass lesion** & $48(22)$ & $40(23)$ & 0.824 \\
\hline $\begin{array}{l}\text { Nonevacuated mass lesion } \\
>25 \mathrm{~cm}^{3} \text { on first CT }\end{array}$ & $3(1)$ & $24(14)$ & $<0.001$ \\
\hline Cortical contusions on CT & $109(50)$ & $93(54)$ & 0.399 \\
\hline In-hospital mortality & $2(1)$ & $51(29)$ & $<0.001$ \\
\hline
\end{tabular}

Pts = patients.

Values are presented as number of patients (\%) or median (IQR) unless otherwise indicated. Boldface type indicates statistical significance.

* There were 7 patients with unknown cause of injury in the nonincluded group.

$\dagger$ Also includes snowboarding.

$\ddagger$ Including 1 gunshot injury in the included group.

$\S$ According to HISS at admission.

I Hypoxia defined as $\mathrm{O}_{2}$ saturation $<92$ and hypotension defined as systolic blood pressure $<90 \mathrm{~mm} \mathrm{Hg}$.

${ }^{* *}$ Evacuated contusion or hematoma at any time point during the hospital stay.

those who fell $<2 \mathrm{~m}(\mathrm{p}=0.078)$. TAI was found in $90 \%$ of those injured in alpine skiing accidents, and in $33 \%$ of victims of violence.

\section{Cortical Contusions and Mass Lesions on CT in Patients With TAI}

Cortical contusions on CT were found in $47 \%$ of patients with TAI, while significant mass lesions were found in 19\%. Cortical contusions and/or mass lesions on CT were found in 53\% of patients with TAI after RTA and $68 \%$ of those with TAI after falls $(\mathrm{p}=0.079$, Table 3, Fig. $3)$. The highest prevalence of contusions and/or mass lesions in patients with TAI was found after falls down the stairs (92\%) and the lowest after alpine accidents (11\%). Mass lesions were more frequent in TAI patients injured in falls than in TAI patients injured in RTAs (36\% vs 9\%, $\mathrm{p}<0.001)$.

\section{Cause of Injury and Grade of TAI on Clinical MRI}

The TAI grades differed significantly between the 4 subcategories of patients injured in RTAs $(\mathrm{p}=0.004$, Table 2). Pedestrians hit by motor vehicle had the overall highest median TAI grade of 3 (82\% TAI grades 2-3, Fig. 4A), car drivers/passengers had a median TAI grade of 2 (76\% TAI grades 2-3), and bicyclists had the lowest median TAI grade of 1 (37\% TAI grades 2-3).

There were also significant differences in TAI grades among the 3 subcategories of patients injured in falls $(\mathrm{p}=$ 0.006). In patients who fell from $>$ own height, the median TAI grade was 1 (45\% TAI grades 2-3, Fig. 4B). Patients who fell down the stairs had a median TAI grade of 1 (26\% TAI grades 2-3), while the lowest median TAI grade of 0 (13\% TAI grades $2-3)$ was found in patients who fell from own height or lower. Fifty percent of patients who fell $>2$ m had TAI grades 2-3, compared to $17 \%$ for those who fell $<2 \mathrm{~m}(\mathrm{p}=0.011)$. Those who suffered an alpine skiing accident had a median TAI grade of 2.5 (70\% TAI grades $2-3$ ). Only 1 of the 3 patients with TAI as a result of violence had TAI grade 2 .

After adjusting for age, GCS score, pupillary dilatation, the presence of cortical contusions, and the presence of mass lesions, TAI grade was a significant predictor for poor outcome (OR 2.78, 95\% CI 1.55-5.48).

\section{Bilateral Traumatic Axonal Injury in the Brainstem and Thalamus}

Twenty-six patients had bilateral TAI lesions in the brainstem $(n=12)$, the thalamus $(n=7)$, or both $(n=7)$. These lesions were found in $17 \%$ of all patients injured in RTAs and in $7 \%$ of patients after falls $(\mathrm{p}=0.043)$. Fifteen of the 19 patients with bilateral lesions after RTAs were car drivers/passengers, 2 were pedestrians, 1 was injured on a motorcycle, and 1 on a bicycle. Four of the 6 falls were from $>2 \mathrm{~m}$, while the remaining 2 were falls down the stairs.

\section{Discussion}

This clinical MRI study of patients with moderate or severe TBI demonstrated a high proportion of patients with TAI grades $2-3$ after causes of injury associated with a high prevalence of TAI. Conversely, a low proportion of grades 2-3 was found in patients after causes of injury associated with a low prevalence of TAI. Pedestrians injured in RTAs had the highest occurrence of TAI and the highest TAI grades of all the injury categories. Next came patients injured in alpine skiing accidents, car drivers/passengers, and patients who fell from $>$ own height. However, TAI was also present among patients who suffered more low-energy trauma: almost two-thirds of patients who fell down the stairs and almost one-third of patients who fell from $\leq$ own height had visible TAI. Importantly, in more than half of all patients with TAI on clinical MRI the CT scan had shown cortical contusions and/or significant mass lesions. 

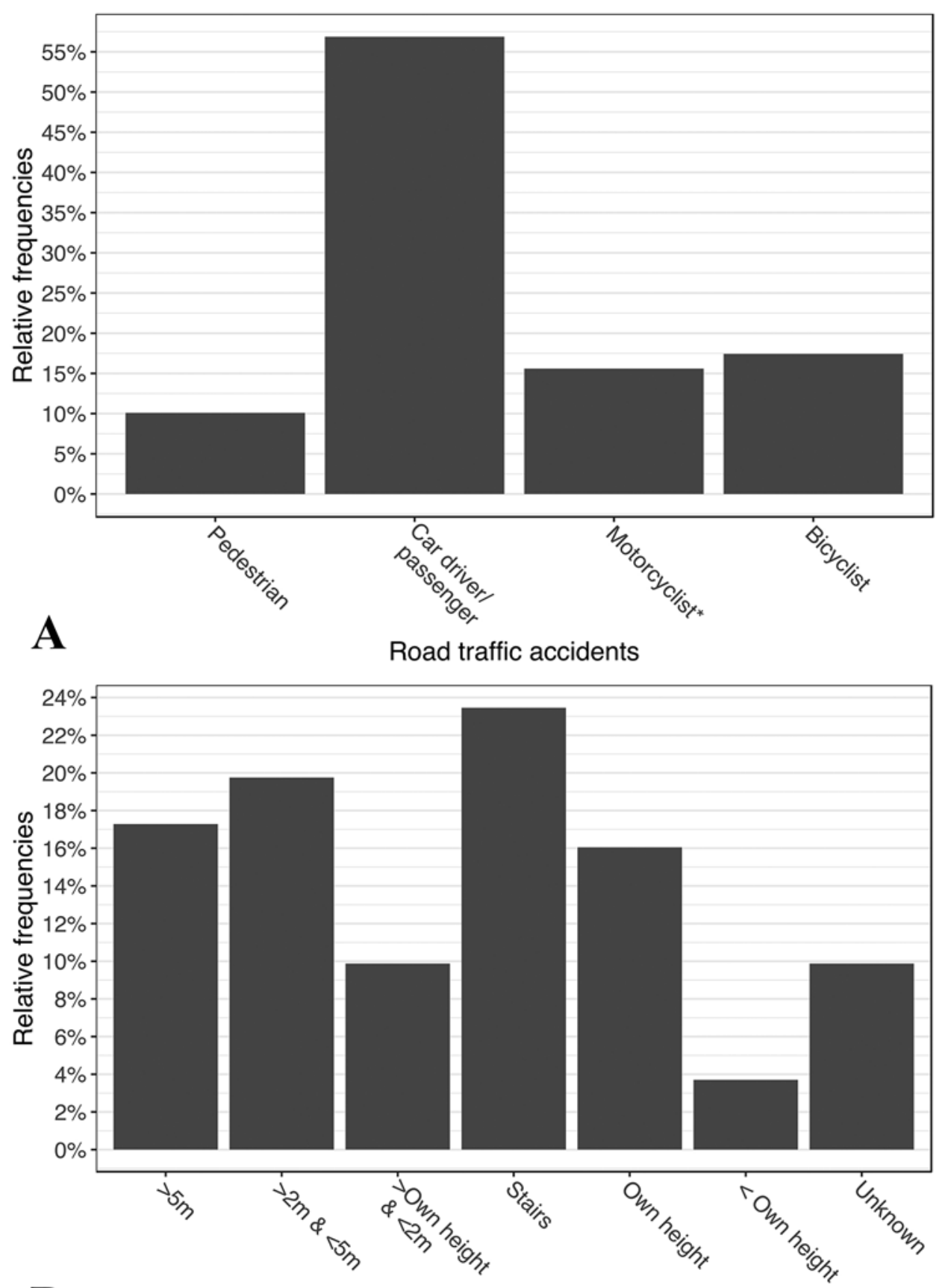

B

Fall injuries

FIG. 2. A: Distribution of patients in the different subcategories of RTAs. All pedestrians were hit by motorized vehicles. *Includes riders of motorcycles, mopeds, and snowmobiles. B: Distribution of patients in the different subcategories of falls.

\section{TAl and RTAs}

TAI was found in the vast majority of patients injured in RTAs. This is in agreement with previous findings that suggest this mode of injury as a special risk factor for TAI. ${ }^{4}$ Pedestrians in RTAs were all hit by a car, and all had TAI. Their TAI grades were overall very high, as illustrated by the high median TAI grade. This is not surprising, as pedestrians are often hit by vehicles with a relatively high speed. The impact will accelerate the body and often throw the pedestrian several meters, leading to an instant deceleration when the head hits the ground. ${ }^{6}$ An autopsy study found histopathological evidence of diffuse TAI, i.e., TAI in at least 3 brain regions and on both sides of the tentorium, ${ }^{9}$ in half of the cases of lethal TBI in pedestrians involved in RTAs. ${ }^{6}$ Similarly, in a large study of hospitalized pediatric TBI patients by Levin et al., over half of all children hit by cars while on foot or on a bicycle had lesions of the white matter or deep gray matter on 
TABLE 2. Cause of injury in relation to the occurrence and grade of TAI and in relation to age, GCS score, and patient outcome in the included patients

\begin{tabular}{lccllll}
\hline \multicolumn{1}{c}{ Cause of Injury } & No. of Pts $(\mathrm{n}=219)$ & TAl & TAl Grade* & Age in Yrs $\dagger$ & GCS Score $\dagger$ & GOSE Score $\dagger$ \\
\hline RTA & 109 & $91(83)$ & $2(1-3)$ & $25(19-42)$ & $7(5-10)$ & $6(5-8)$ \\
\hline Pedestrian & 11 & $11(100)$ & $3(2-3)$ & $18(14-23)$ & $6(6-8)$ & $6(5.5-7.5)$ \\
\hline Car driver/passenger & 62 & $55(89)$ & $2(2-3)$ & $24(19-34)$ & $6.5(4-8.3)$ & $6(4.8-8)$ \\
\hline Motorcyclist & 17 & $12(71)$ & $1(0-3)$ & $33(23-45)$ & $10(7.8-12.2)$ & $8(5.5-8)$ \\
\hline Bicyclist & 19 & $13(68)$ & $1(0-2)$ & $42(30-57)$ & $9(8-12)$ & $6(6-8)$ \\
\hline Fall & 81 & $50(62)$ & $1(0-2)$ & $33(23-53)$ & $8(5-13)$ & $6(4-8)$ \\
\hline$>$ Own height & 38 & $29(76)$ & $1(1-2)$ & $30(23-52)$ & $8(5.8-13)$ & $6.5(3.8-8)$ \\
\hline Down the stairs & 19 & $12(63)$ & $1(0-1.5)$ & $36(25-50)$ & $4(3-11)$ & $5(4-6.5)$ \\
\hline SOwn height & 16 & $5(31)$ & $0(0-1)$ & $52(39-60)$ & $12.5(11.8-13)$ & $6(6-8)$ \\
\hline Unknown fall height & 8 & $4(50)$ & $0.5(0-2)$ & $26(13-45)$ & $9(8-11)$ & $8(6.5-8)$ \\
\hline Alpine skiing accident & 10 & $9(90)$ & $2.5(1.3-3)$ & $16(15-21)$ & $11(9-11)$ & $8(7-8)$ \\
\hline Violence & 9 & $3(33)$ & $0(0-1)$ & $42(31-53)$ & $12.5(12.2-12.8)$ & $8(6.5-8)$ \\
\hline Other & 10 & $8(80)$ & $1(1-2)$ & $35(14-50)$ & $10(8.5-12)$ & $7(5.8-8)$ \\
\hline
\end{tabular}

Values are presented as number of patients (\%) or median (IQR) unless otherwise indicated.

* The absence of TAI was analyzed as TAI grade 0 .

$\dagger$ Among patients with TAl.

$\ddagger$ Includes riders of motorcycles, mopeds, and snowmobiles.

MRI in the chronic phase of TBI (ranging from 3 months to several years postinjury). ${ }^{20}$ Although Levin et al. did not study TAI lesions specifically, both of these studies support the current findings of pedestrians in RTAs as a group that is particularly vulnerable to severe TAI.

Car drivers and passengers represented the largest group of patients in the RTA category, and they had both

TABLE 3. CT findings of cortical contusions and mass lesions by cause of injury in the 161 included patients with TAI

\begin{tabular}{|c|c|c|c|c|}
\hline Cause of Injury & $\begin{array}{l}\text { No. } \\
\text { of } \\
\text { Pts }\end{array}$ & $\begin{array}{l}\text { No. of Pts } \\
\text { w/ Cortical } \\
\text { Contusions }\end{array}$ & $\begin{array}{c}\text { No. of } \\
\text { Pts w/ } \\
\text { Mass } \\
\text { Lesions* }\end{array}$ & $\begin{array}{l}\text { Total No. of } \\
\text { Pts w/ Cortical } \\
\text { Contusions } \\
\text { \&/or Mass } \\
\text { Lesions* }\end{array}$ \\
\hline RTA & 91 & $43(47)$ & $8(9)$ & $48(53)$ \\
\hline Pedestrian & 11 & $5(46)$ & $1(9)$ & $6(55)$ \\
\hline Car driver/passenger & 55 & $31(56)$ & $4(7)$ & $33(60)$ \\
\hline Motorcyclist $†$ & 12 & $2(17)$ & $1(8)$ & $3(25)$ \\
\hline Bicyclist & 13 & $5(39)$ & $2(15)$ & $6(46)$ \\
\hline Fall & 50 & $28(56)$ & $18(36)$ & $34(68)$ \\
\hline$>$ Own height & 29 & $15(52)$ & $7(24)$ & $18(62)$ \\
\hline Down the stairs & 12 & $8(67)$ & $9(75)$ & $11(92)$ \\
\hline$\leq$ Own height & 5 & $3(60)$ & $1(20)$ & $3(60)$ \\
\hline Unknown fall height & 4 & $2(50)$ & $1(25)$ & $2(50)$ \\
\hline Alpine skiing accident & 9 & $1(11)$ & $0(0)$ & $1(11)$ \\
\hline Violence & 3 & $1(33)$ & $2(67)$ & $2(67)$ \\
\hline Total & 161 & $76(47)$ & $30(19)$ & $88(55)$ \\
\hline
\end{tabular}

a high prevalence of TAI and high TAI grades. The lack of studies examining the association between cause of injury and TAI on MRI in the early phase makes it difficult to compare the current results with previous findings. The results of the current study are, on the other hand, in line with what was found in car drivers and passengers in an autopsy study of TBI patients. ${ }^{7}$ Also, the high TAI grades found in this group of patients are not surprising, considering the forces often involved in car accidents.

The lowest percentage of TAI and the lowest TAI grades in the RTA category were found among bicyclists. Still, as many as $60 \%$ of these patients had TAI. Bicycle accidents typically involve the patient flying over the handlebars and hitting the ground at a relatively high speed. In contrast to the current findings, Davceva et al. reported that bicyclists more often had acute subdural hematomas as opposed to TAI ${ }^{6}$ However, the majority of patients with TBI survive, making results from autopsy studies less generalizable than those from clinical MRI studies. Also, whereas MRI examines the entire brain, histopathological examination is usually restricted to a selection of slices from certain brain regions.

\section{TAl and Falls}

Patients injured in falls had a significantly lower prevalence of TAI compared to those injured in RTAs. Still, $62 \%$ of patients had TAI after falling injuries, and the rate was as high as $73 \%$ in those patients who fell from above $2 \mathrm{~m}$. Also, half of the patients in the latter group had TAI grades 2-3. Moreover, approximately two-thirds of patients who fell down the stairs had TAI, almost all of whom also had contusions or mass lesions on CT. Two of these patients even had bilateral TAI lesions in the brainstem and/or thalamus. The current study also found that patients with moderate or severe TBI who fell from $\leq$ own height, which is considered a low-energy injury, had vis- 

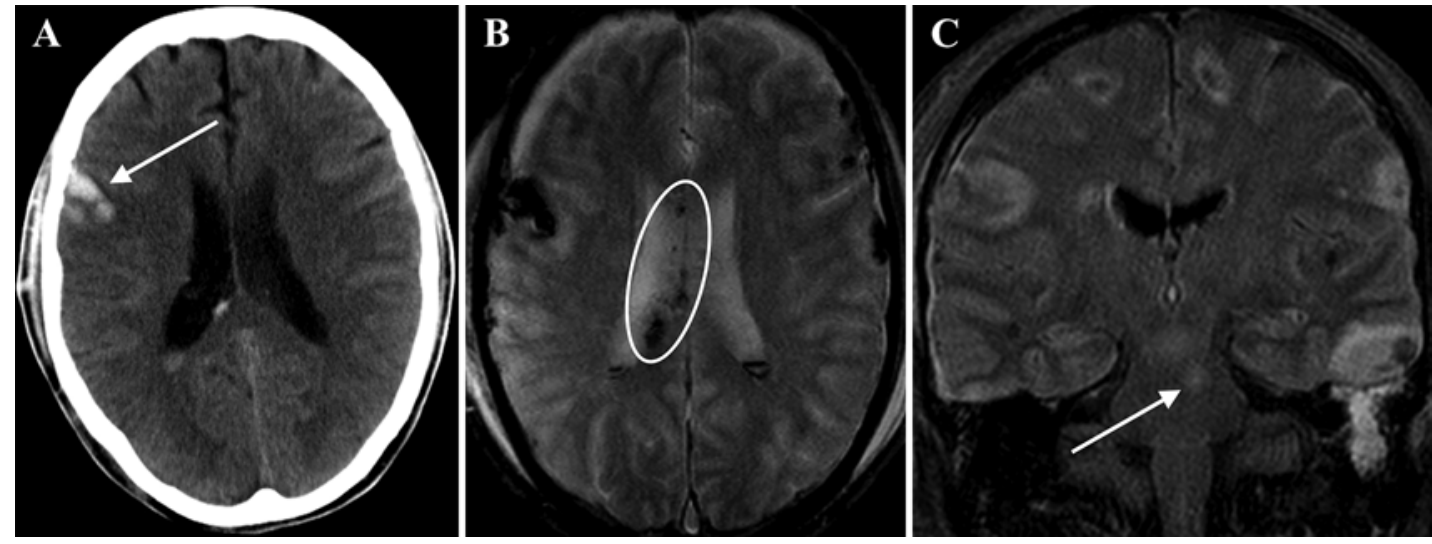

FIG. 3. Imaging of a patient with severe TBI after falling down the stairs. A: CT scan showing cortical contusion (arrow), as well as intraventricular blood in the posterior horn of the right lateral ventricle. B: T2* GRE MR image showing TAl lesions in the corpus callosum (encircled). Intraventricular blood can be seen in the posterior horns of both lateral ventricles. C: FLAIR MR image showing TAl in the brainstem (arrow).

ible TAI lesions in $31 \%$ of the cases. This is in contrast to autopsy studies, some of which have concluded that this type of trauma has insufficient duration of the acceleration forces on the brain to cause TAI., 3,6,9 Then again, as previously discussed, findings from autopsy studies might not be comparable to those from MRI studies. It is important to note, however, that most patients who suffer such low-energy injuries do not sustain moderate to severe, but rather mild TBI. In fact, such falls were a rare cause (only $1 \%$ from < own height and $6 \%$ from own height) of moderate or severe TBI in our cohort.

\section{TAl and Other Causes of Injury}

In this study, $90 \%$ of patients who suffered alpine skiing accidents had TAI lesions, and 4 out of 5 had TAI grades $2-3$. These results suggest that alpine skiing and snowboarding accidents might be more comparable to RTAs than to falls in terms of TAI severity. However, compared to other causes of injury associated with high TAI grades, very few of the patients with TAI due to alpine skiing accidents had cortical contusions and/or mass lesions on CT.

We also found that 3 of the 9 patients who were victims of violence had TAI. Results of some autopsy studies have been reported to indicate that TAI after assault is a rare occurrence, ${ }^{6}$ but this would depend on the definition of TAI, the staining methods used, ${ }^{9}$ and the type of violence. Our findings are in line with the report of both histopathologically diffuse TAI in $30 \%$ of victims of fatal TBIs after assault, ${ }^{14}$ and TAI on clinical MRI in $29 \%$ of 100 professional boxers and martial arts fighters. ${ }^{29}$ Altogether, these findings support the notion that TAI may be a not uncommon consequence of blunt violence.

\section{CT Findings of Contusions and Mass Lesions in Patients With TAI}

Cortical contusions on CT were demonstrated in ap-
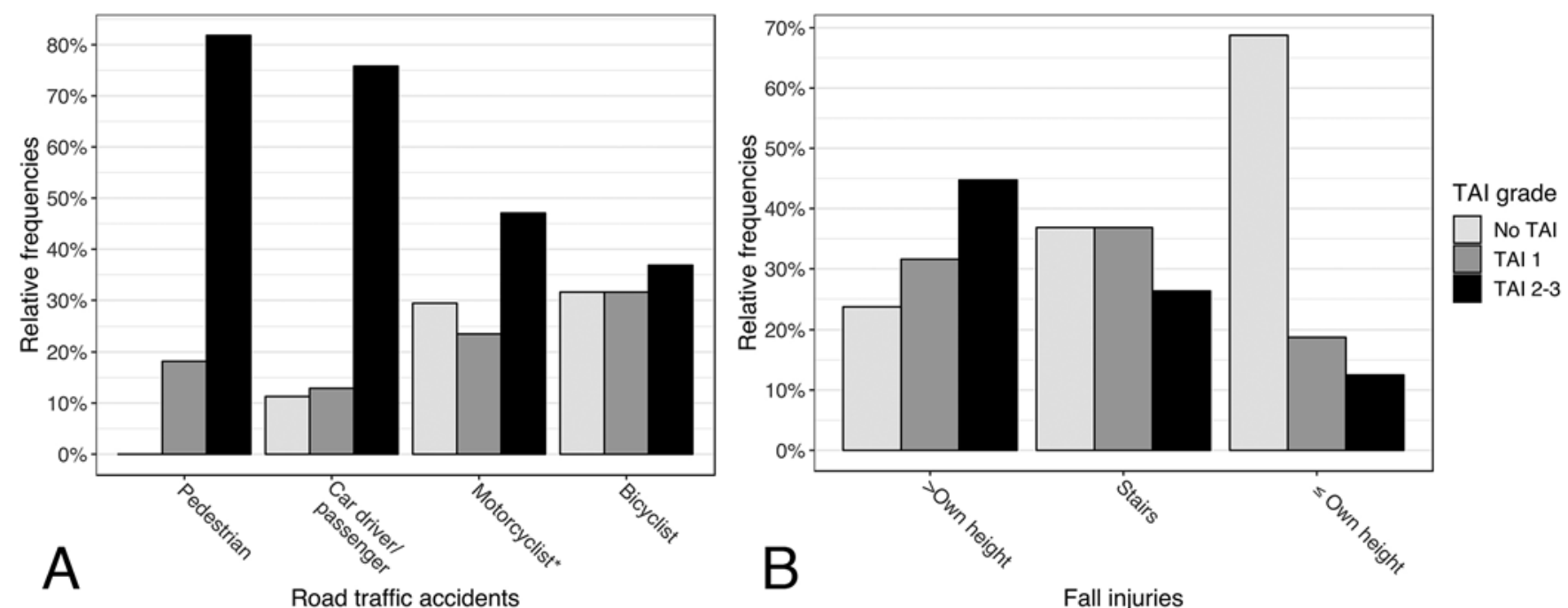

FIG. 4. A: Relative frequencies of grade of TAI in patients involved in different types of RTAs. *Encompasses riders of motorcycles, mopeds, and snowmobiles. B: Frequencies of grade of TAI relative to fall height in patients involved in fall accidents. 
proximately half of the patients with TAI after RTAs and falls. Mass lesions occurred more often after falls than after RTAs in patients with TAI. Rather than causing TAI, falls are commonly thought to result in cortical contusions, intracranial hematomas, or skull fractures. However, as our results show, such lesions can-and often do-occur alongside of each other. Moreover, TAI grade was a significant predictor of poor outcome after adjusting for CT findings and known clinical predictors. These findings may be of importance for physicians, who might not consider the possibility of TAI after a fall if the patient has CT findings of cortical contusions or mass lesions.

\section{Bilateral Lesions in the Brainstem and Thalamus}

Almost three-quarters of the patients with bilateral TAIs in the brainstem or thalamus were injured in RTAs. The vast majority of these were injured in car accidents. The patients who had bilateral TAI in these structures after falls had fallen either from above $2 \mathrm{~m}$ or down the stairs. Hence, such MRI findings, which are related to both the GCS score in the acute phase and patient outcome, ${ }^{24,32}$ were for the most part found in the subcategories of RTA and fall associated with a high prevalence and grade of TAI. Lesions in central and deep parts of the brain would indeed indicate high-energy trauma, ${ }^{28}$ and bilateral TAIs in the brainstem and/or thalamus are suggested to possibly be a separate and worst grade of TAI in a new TAI classification. $^{24}$

\section{Study Strengths and Limitations}

The size of this 10-year TBI cohort and the prospective nature of the data collection are notable strengths of this study. Also, there were no differences in the cause of injury between patients included in the present MRI study and the nonincluded patients. Furthermore, MRI readings were conducted blinded for clinical information, and interrater reliability tests were performed in an earlier study. ${ }^{25}$

The current study had some limitations. First, there were some differences between the included and the nonincluded patients. However, there will always be a selection bias to MRI studies, because the patients that die in the acute phase (29\% of the nonincluded patients in this study) are typically not examined by MRI. Second, the time from injury to MRI varied across patients. However, a study from our research group of TBI patients receiving repeated MRI showed that TAI lesions on T2*GRE persist after 3 months postinjury. ${ }^{25}$ Also, the fact that as many as $75 \%$ of patients had an MRI scan within 17 days of injury and that patients with MRI later than 5 weeks postinjury were excluded would improve the detection rate for lesions on FLAIR and DWI that tend to attenuate over time. ${ }^{25}$ Third, most of the MR images were acquired at 1.5 $\mathrm{T}$, and our harmonized MRI protocol for the most part did not include SWI. Both 3-T scanning ${ }^{31}$ and SWI ${ }^{1,13}$ increase the sensitivity for lesion detection, which could possibly have led to both higher occurrence and grades of TAI. On the other hand, a recent study from our research group using both 3-T scanning and SWI within 72 hours found TAI lesions in only $6 \%$ of patients with mild TBI, ${ }^{8}$ compared to $74 \%$ of patients with moderate and severe $\mathrm{TBI}$ in the current study. Hence, 1.5-T scanning and T2*GRE may be sufficiently sensitive to detect visible TAI lesions in moderate and severe TBI. ${ }^{31}$

\section{Conclusions}

This prospective study of patients with moderate or severe TBI is to our knowledge the first clinical MRI study to clearly demonstrate both high prevalence and grade of TAI after most of the different types of RTAs, alpine skiing accidents, and falls from a height. However, TAI was also found in patients following more low-energy trauma such as falls down the stairs or from their own height.

Furthermore, TAI grade was associated with outcome, and physicians managing TBI patients in the acute phase should be aware of the possibility of TAI no matter the cause of injury and also when the CT scan shows cortical contusions or mass lesions.

\section{Acknowledgments}

We thank Jian Xu (J.X.), Kjell Arne Kvistad (K.A.K.), Jana Rydland (J.R.), and Mari Folvik (M.F.) for their contributions in the MRI readings. We also thank Stine Borgen Lund, Oddrun Sandrød, Otto Aarhaug, and Beate Mærk Voll for their management of the patient database.

H.K.M. and K.G.M. received funding from the Norwegian University of Science and Technology (NTNU), ERA-NET NEURON, and the Research Council of Norway (TAI-MRI project). T.S. received a research grant during the study period from the liaison committee between the Central Norway regional health authority and NTNU.

\section{References}

1. Abu Hamdeh S, Marklund N, Lannsjö M, Howells T, Raininko R, Wikström J, et al: Extended anatomical grading in diffuse axonal injury using MRI: hemorrhagic lesions in the substantia nigra and mesencephalic tegmentum indicate poor long-term outcome. J Neurotrauma 34:341-352, 2017

2. Adams JH, Doyle D, Ford I, Gennarelli TA, Graham DI, McLellan DR: Diffuse axonal injury in head injury: definition, diagnosis and grading. Histopathology 15:49-59, 1989

3. Adams JH, Doyle D, Graham DI, Lawrence AE, McLellan DR: Diffuse axonal injury in head injuries caused by a fall. Lancet 2:1420-1422, 1984

4. Adams JH, Graham DI, Murray LS, Scott G: Diffuse axonal injury due to nonmissile head injury in humans: an analysis of 45 cases. Ann Neurol 12:557-563, 1982

5. Cicuendez M, Castaño-León A, Ramos A, Hilario A, Gómez PA, Lagares A: The added prognostic value of magnetic resonance imaging in traumatic brain injury: the importance of traumatic axonal injury when performing ordinal logistic regression. J Neuroradiol 46:299-306, 2019

6. Davceva N, Janevska V, Ilievski B, Petrushevska G, Popeska $\mathrm{Z}$ : The occurrence of acute subdural haematoma and diffuse axonal injury as two typical acceleration injuries. J Forensic Leg Med 19:480-484, 2012

7. Davceva N, Sivevski A, Basheska N: Traumatic axonal injury, a clinical-pathological correlation. J Forensic Leg Med 48:35-40, 2017

8. Einarsen CE, Moen KG, Håberg AK, Eikenes L, Kvistad KA, $\mathrm{Xu}$ J, et al: Patients with mild traumatic brain injury recruited from both hospital and primary care settings: a controlled longitudinal MRI study. J Neurotrauma [epub ahead of print], 2019

9. Geddes JF, Whitwell HL, Graham DI: Traumatic axonal 
injury: practical issues for diagnosis in medicolegal cases. Neuropathol Appl Neurobiol 26:105-116, 2000

10. Gennarelli TA, Thibault LE, Adams JH, Graham DI, Thompson CJ, Marcincin RP: Diffuse axonal injury and traumatic coma in the primate. Ann Neurol 12:564-574, 1982

11. Gentry LR: Imaging of closed head injury. Radiology 191:117,1994

12. Gentry LR, Godersky JC, Thompson B: MR imaging of head trauma: review of the distribution and radiopathologic features of traumatic lesions. AJR Am J Roentgenol 150:663672,1988

13. Geurts BHJ, Andriessen TMJC, Goraj BM, Vos PE: The reliability of magnetic resonance imaging in traumatic brain injury lesion detection. Brain Inj 26:1439-1450, 2012

14. Graham DI, Clark JC, Adams JH, Gennarelli TA: Diffuse axonal injury caused by assault. J Clin Pathol 45:840-841, 1992

15. Håberg AK, Olsen A, Moen KG, Schirmer-Mikalsen K, Visser E, Finnanger TG, et al: White matter microstructure in chronic moderate-to-severe traumatic brain injury: Impact of acute-phase injury-related variables and associations with outcome measures. J Neurosci Res 93:1109-1126, 2015

16. Hill CS, Coleman MP, Menon DK: Traumatic axonal injury: mechanisms and translational opportunities. Trends Neurosci 39:311-324, 2016

17. Inglese M, Makani S, Johnson G, Cohen BA, Silver JA, Gonen O, et al: Diffuse axonal injury in mild traumatic brain injury: a diffusion tensor imaging study. J Neurosurg 103:298-303, 2005

18. Jennett B, Snoek J, Bond MR, Brooks N: Disability after severe head injury: observations on the use of the Glasgow Outcome Scale. J Neurol Neurosurg Psychiatry 44:285293, 1981

19. Johnson VE, Stewart W, Smith DH: Axonal pathology in traumatic brain injury. Exp Neurol 246:35-43, 2013

20. Levin HS, Mendelsohn D, Lilly MA, Yeakley J, Song J, Scheibel RS, et al: Magnetic resonance imaging in relation to functional outcome of pediatric closed head injury: a test of the Ommaya-Gennarelli model. Neurosurgery 40:432-441, 1997

21. Maas AIR, Steyerberg EW, Butcher I, Dammers R, Lu J, Marmarou A, et al: Prognostic value of computerized tomography scan characteristics in traumatic brain injury: results from the IMPACT study. J Neurotrauma 24:303-314, 2007

22. Makino Y, Arai N, Hoshioka Y, Yoshida M, Kojima M, Horikoshi $\mathrm{T}$, et al: Traumatic axonal injury revealed by postmortem magnetic resonance imaging: a case report. Leg Med (Tokyo) 36:9-16, 2019

23. Marshall LF, Marshall SB, Klauber MR, Marjan van Berkum C, Eisenberg HM, Jane JA, et al: A new classification of head injury based on computerized tomography. J Neurosurg 75 Suppl:S14-S20, 1991

24. Moe HK, Moen KG, Skandsen T, Kvistad KA, Laureys S, Håberg A, et al: The influence of traumatic axonal injury in thalamus and brainstem on level of consciousness at scene or admission: a clinical magnetic resonance imaging study. $\mathbf{J}$ Neurotrauma 35:975-984, 2018

25. Moen KG, Skandsen T, Folvik M, Brezova V, Kvistad KA, Rydland J, et al: A longitudinal MRI study of traumatic axonal injury in patients with moderate and severe traumatic brain injury. J Neurol Neurosurg Psychiatry 83:1193-1200, 2012

26. Moen KG, Vik A, Olsen A, Skandsen T, Håberg AK, Evensen KAI, et al: Traumatic axonal injury: Relationships between lesions in the early phase and diffusion tensor imaging parameters in the chronic phase of traumatic brain injury. J Neurosci Res 94:623-635, 2016

27. Murray JG, Gean AD, Evans SJJ: Imaging of acute head injury. Semin Ultrasound CT MR 17:185-205, 1996
28. Ommaya AK, Gennarelli TA: Cerebral concussion and traumatic unconsciousness. Correlation of experimental and clinical observations of blunt head injuries. Brain 97:633-654, 1974

29. Orrison WW, Hanson EH, Alamo T, Watson D, Sharma M, Perkins TG, et al: Traumatic brain injury: a review and highfield MRI findings in 100 unarmed combatants using a literature-based checklist approach. J Neurotrauma 26:689-701, 2009

30. Parizel PM, Van Goethem JW, Özsarlak O, Maes M, Phillips CD: New developments in the neuroradiological diagnosis of craniocerebral trauma. Eur Radiol 15:569-581, 2005

31. Scheid R, Ott DV, Roth H, Schroeter ML, von Cramon DY: Comparative magnetic resonance imaging at 1.5 and 3 Tesla for the evaluation of traumatic microbleeds. J Neurotrauma 24:1811-1816, 2007

32. Skandsen T, Kvistad KA, Solheim O, Lydersen S, Strand IH, Vik A: Prognostic value of magnetic resonance imaging in moderate and severe head injury: a prospective study of early MRI findings and one-year outcome. J Neurotrauma 28:691-699, 2011

33. Skandsen T, Kvistad KA, Solheim O, Strand IH, Folvik M, Vik A: Prevalence and impact of diffuse axonal injury in patients with moderate and severe head injury: a cohort study of early magnetic resonance imaging findings and 1-year outcome. J Neurosurg 113:556-563, 2010

34. Stein SC, Spettell C: The Head Injury Severity Scale (HISS): a practical classification of closed-head injury. Brain Inj 9:437-444, 1995

35. Toth A, Kornyei B, Kovacs N, Rostas T, Buki A, Doczi T, et al: Both hemorrhagic and non-hemorrhagic traumatic MRI lesions are associated with the microstructural damage of the normal appearing white matter. Behav Brain Res 340:106116,2018

36. Weiss N, Galanaud D, Carpentier A, Naccache L, Puybasset L: Clinical review: Prognostic value of magnetic resonance imaging in acute brain injury and coma. Crit Care 11:230, 2007

\section{Disclosures}

The authors report no conflict of interest concerning the materials or methods used in this study or the findings specified in this paper.

\section{Author Contributions}

Conception and design: Moe, Limandvik Myhr, Vik. Acquisition of data: Limandvik Myhr, Moen, Skandsen, Vik. Analysis and interpretation of data: all authors. Drafting the article: Moe, Limandvik Myhr, Vik. Critically revising the article: all authors. Approved the final version of the manuscript on behalf of all authors: Moe. Statistical analysis: Moe, Limandvik Myhr. Administrative/technical/material support: Moe. Study supervision: Vik.

\section{Supplemental Information \\ Previous Presentations}

Portions of this work were presented in poster form at the Third Joint Symposium of the National and International Neurotrauma Societies and the AANS/CNS Section on Neurotrauma and Critical Care, Toronto, Canada, August 11-16, 2018. The conference abstracts were also published online (DOI: 10.1089/ neu.2018.29013.abstracts).

\section{Correspondence}

Hans Kristian Moe: Norwegian University of Science and Technology, Trondheim, Norway. hanskristianmoe91@gmail.com. 